\title{
Impact of Vaccination on the World Epizootic Situation Plague of Small Ruminants
}

\author{
Larisa Padilo, Valery Agoltsov, and Oksana Biryukova* \\ Saratov State Agrarian University named after N.I. Vavilov, Saratov, 410012, 1 Teatralnaya \\ Ploshchad, Russian Federation
}

\begin{abstract}
According to OIE and FAO data in 2009-2019, there have been 2,884 outbreaks of plague of small ruminants (PPR) worldwide, mainly in Africa and Asia. Mortality from this pathology reaches $100 \%$. The economic damage caused by this disease to animal husbandry consists of a mass mortality, a decrease in the productivity of sick animals and the cost of quarantine measures in a disadvantaged area. Cartographic analysis showed that the highest concentration of PPR outbreaks for the indicated period was observed in five main regions. Northwest Africa (includes Morocco, Algeria and Tunisia); The Black Sea coast of Bulgaria; Eastern coast of the Mediterranean Sea (Israel); People's Republic of Bangladesh; Southern, Southeastern and Eastern parts of the People's Republic of China. Outbreaks with the largest number of dead animals were concentrated mainly in Bangladesh and China. Outbreaks with the largest number of dead animals were concentrated mainly in Bangladesh and China, where vaccinations have not been carried out in recent years. The study of the correlation between the PPR incidence rate and the number of animals immunized in the most disadvantaged states found only modest reductions in incidence as a result of vaccination campaigns. Pearson's correlation coefficient was - 0.13 for Algeria, - 0.19 for Bangladesh and 0.33 for Israel, which appears to be due to the small scale of vaccination campaigns. The obtained results of the epizootic analysis by PPR indicate insufficient coverage of animals susceptible to the pathogen by vaccination and a greater likelihood of new outbreaks of the disease not only in disadvantaged countries, but also the spread of infection in new territories.
\end{abstract}

\section{Introduction}

Plague of small ruminants (PPR) is an acute infectious disease characterized by high contagiousness, the causative agent of which is an RNA-containing virus of the Morbillivirus genus of the Paramyxoviridae family. Goats and sheep, as well as wild small

\footnotetext{
* Corresponding author: kras-bi@yandex.ru
} 
ruminants are susceptible to PPR. Clinical signs of PPR are fever, serous discharge from the eyes and nose, diarrhea, and pneumonia. Infection occurs through direct contact of healthy animals with sick, aerogenic and alimentary routes. Young individuals are most susceptible to this infection [10].

PPR is one of the main causes of devastating losses in the small-scale livestock industry in disease-disadvantaged regions of the world. Mortality from this pathology reaches $100 \%$. The economic damage caused by this disease to animal husbandry consists of a mass mortality, a decrease in the productivity of sick animals and the cost of quarantine measures in a disadvantaged area $[8 ; 11 ; 4 ; 5]$.

To prevent PPR damage, susceptible flocks are vaccinated. A number of vaccines based on attenuated PPRV strains are currently in use [1]. The most commonly used strains for production of vaccines are Nigeria/75/1 and Sungri/96 [3]. Vaccinated sheep and goats acquire active lifelong immunity against disease [12].

Despite this, the world continues to register up to several thousand cases of PPR per year [3].

Due to the existing epizootic distress, the purpose of our work was to study the impact of ongoing vaccination campaigns on the epizootic situation according to PPR.

\section{Methods}

When analyzing the epizootic situation and evaluating the effectiveness of vaccination campaigns for small ruminants, we used information from the International Database of the World Organization for Animal Health (OIE) WAHIS [13] and the World Animal Diseases Information System of the Food and Agriculture Organization (FAO) UN EMPRES- i [7].

To study the spatial distribution of PPR breakouts, we used geospatial analysis procedures from the ESRI ArcGIS Desktop geographic information system. The visualization of clusters of breakouts (clusters) was carried out using the GIS-procedure "Density of nuclei", which allows converting an array of point information (spatial coordinates of breakouts) into a complex of continuous data by extrapolation $[10 ; 8]$.

The study of the effectiveness of vaccination campaigns was carried out by assessing the dependence of the incidence rate of the disease in the territory of individual states and the proportion of immunized animals, calculating the Pearson correlation coefficient.

The data were statistically processed in Excel from the Microsoft Office 2016 package.

\section{Results}

According to OIE and FAO data in 2009-2019 in the world there were 2884 outbreaks of plague of small ruminants, located mainly in Africa and Asia.

The conducted geospatial analysis showed that the highest concentration of PPR breakouts for the specified period was observed in five large clusters (Figure 1):

- Western part of the northern coast of Africa, (Algeria and Tunisia);

- Western part of the Black Sea coast (Bulgaria);

— Eastern coast of the Mediterranean Sea (Israel);

- People's Republic of Bangladesh;

— Southern, Southeastern and Eastern parts of the People's Republic of China. 


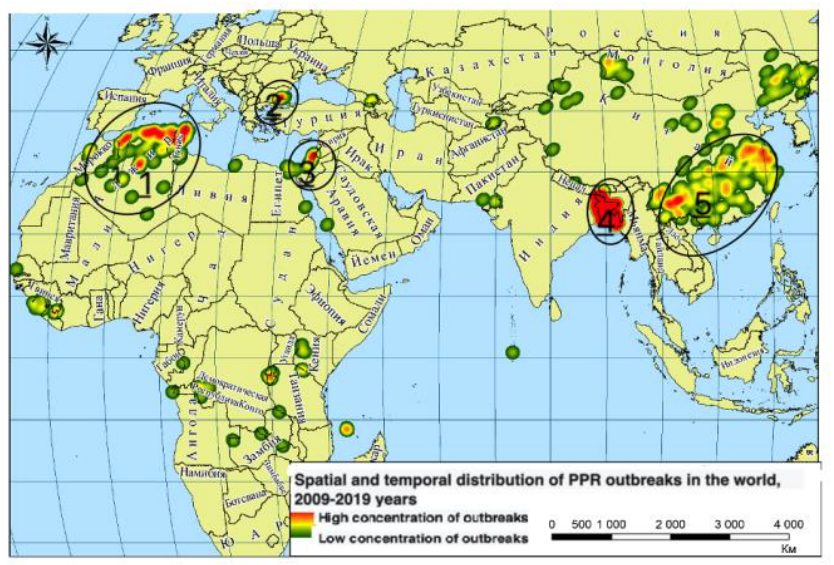

Fig. 1. Spatial-temporal distribution of PPR outbreaks in the world, 2009-2019. Clusters: 1 - North African zone (Morocco, Algeria, Tunisia); 2 - Bulgaria; 3 - Palestine; 4- Bangladesh, 5 - China.

The calculation of the stationarity index showed that the longest disadvantage in terms of PPR was in China, Algeria, Israel, Tunisia and Bangladesh (Figure 2). The same countries also recorded the largest number of outbreaks during this period (Figure 3 ).

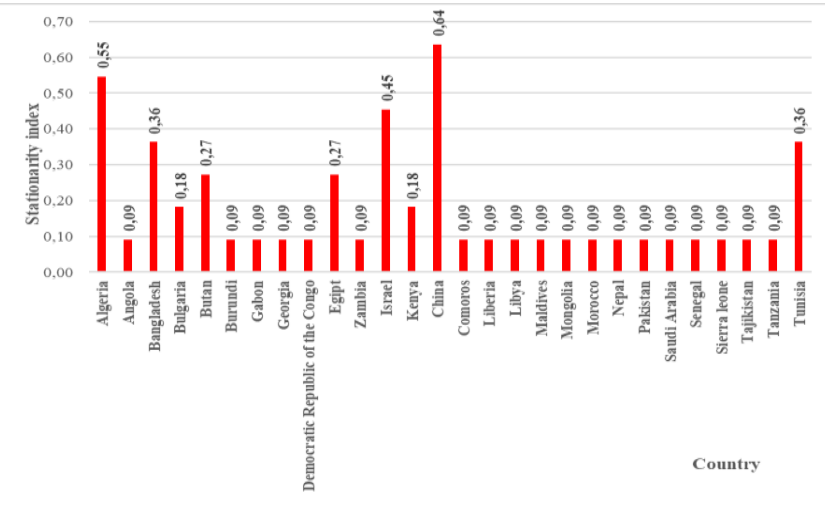

Fig. 2. Index of PPR stationarity in the world, 2009-2019.

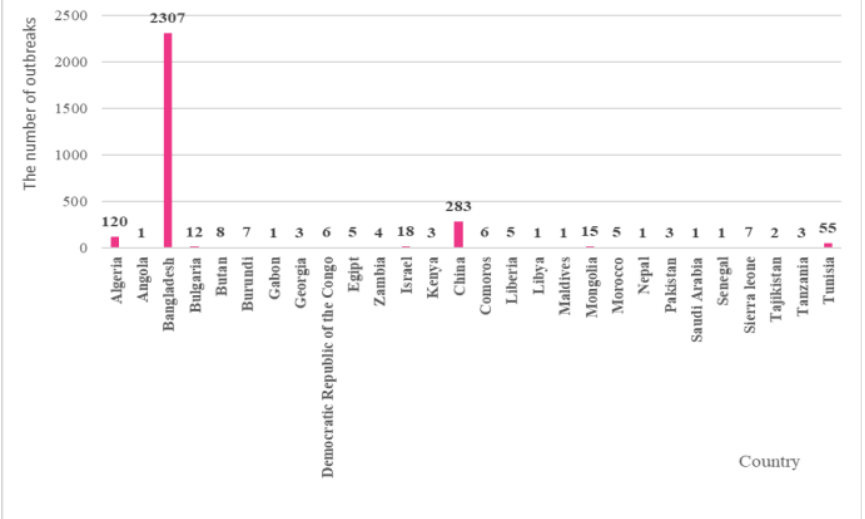


Fig. 3. The number of outbreaks of PPR in different states for 2009-2019.

Wherein, the outbreaks with the largest number of dead animals were concentrated mainly in Bangladesh and China (Figure 4).

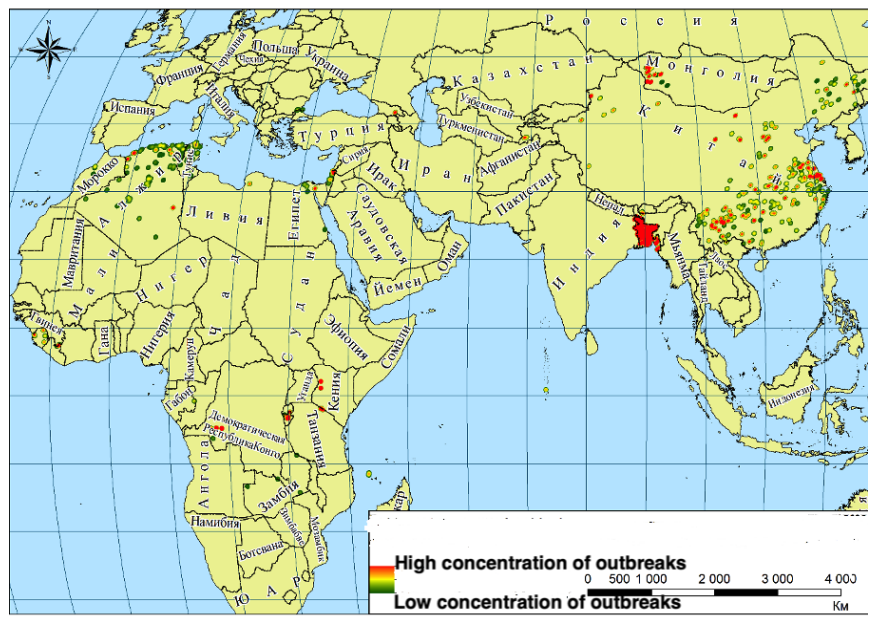

Fig. 4. Spatial-temporal distribution of PPR outbreaks with the highest number of dead animals in the world, 2009-2019.

To assess the impact of ongoing vaccination campaigns on the epizootic situation according to PPR, a comparison was made between the number of vaccinated livestock of susceptible farm animals and the incidence of the disease in the countries with the greatest disadvantage (Figure 5).

According to the OIE, during the period under review, no country in the world had vaccination campaigns covering $100 \%$ of the small ruminant population. In the countries most disadvantaged in terms of PPR, the proportion of vaccinated livestock did not exceed $5.5 \%$. The longest preventive measures were in Israel (2009 - 2014), however, the share of newly vaccinated livestock did not exceed $1.5 \%$ of the total number of susceptible farm animals. Bangladesh achieved the highest vaccination rate during the annual campaign: $5.18 \%$ in 2009, after which no vaccinations were carried out. In Algeria, the corresponding measures against PPR were carried out in 2013, but the share of vaccinated livestock was no more than $0.38 \%$. China and Tunisia did not conduct vaccination campaigns during the reporting period. 


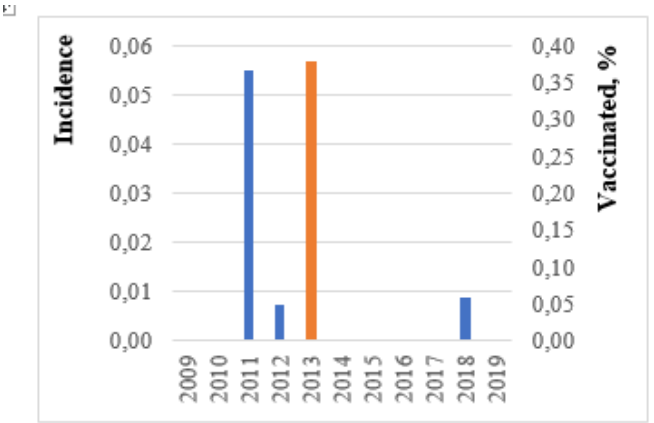

Algeria

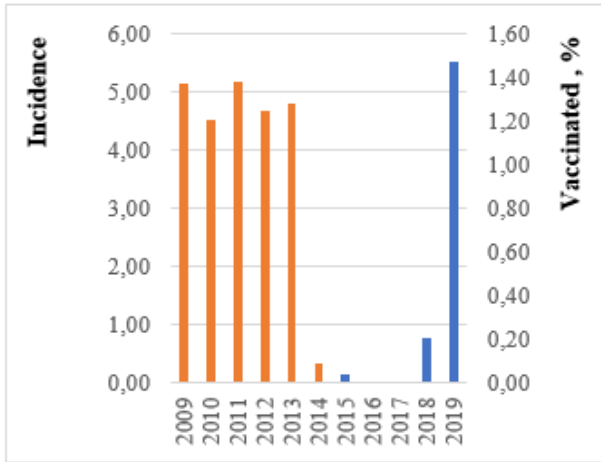

Israel|

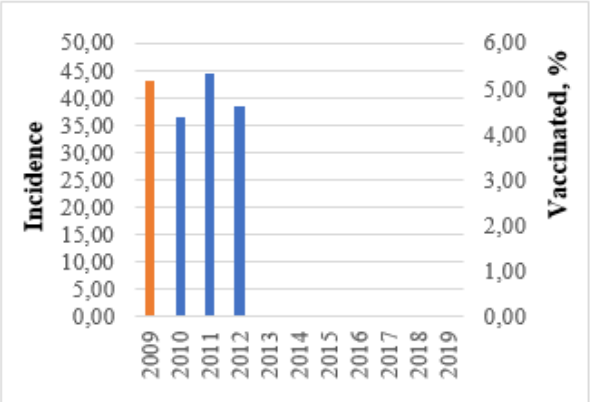

Bangladesh

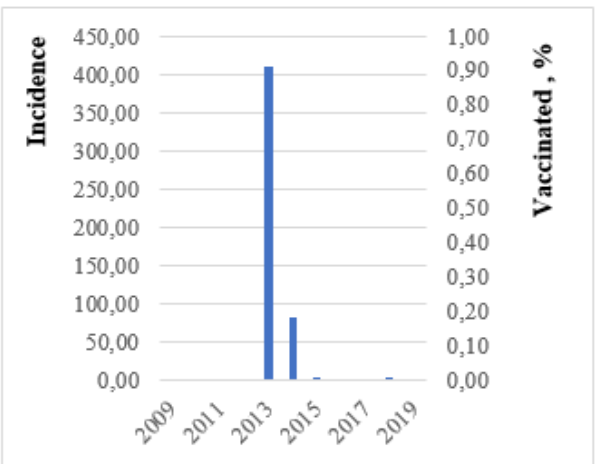

China

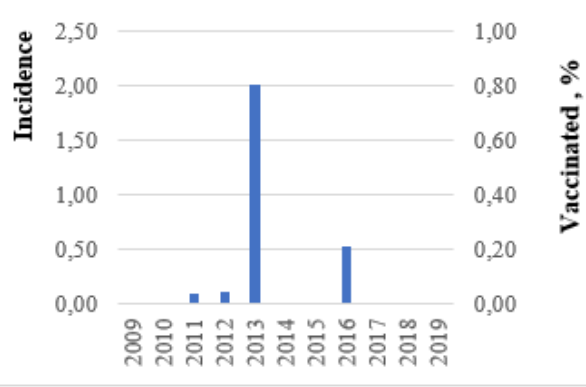

Tunisia

Fig. 5. Incidence of PPR and the number of small ruminants vaccinated against $P P R$ in countries with the worst epizootic situation, 2009-2019. (blue indicates incidence, red -\% of the vaccinated population).

\section{Discussion}

The study of the correlation between the PPR incidence rate and the number of animals immunized in the most disadvantaged states found only modest reductions in incidence as a result of vaccination campaigns. Pearson's correlation coefficient was - 0.13 for Algeria, 0.19 for Bangladesh and -0.33 for Israel, which appears to be due to the small scale of vaccination campaigns.

\section{Conclusion}


Therefore, we can conclude that for the period from 2009 to 2019, in the world there were 2884 outbreaks of plague of small ruminants. All outbreaks were recorded in the Old World, mainly in Africa and Asia.

Cartographic analysis showed that the highest concentration of PPR outbreaks for the indicated period was observed in five main regions. First of all, this is the North-Western part of Africa: (includes Morocco, Algeria and Tunisia), as well as the Black Sea coast of Bulgaria; Eastern coast of the Mediterranean Sea (Israel); People's Republic of Bangladesh; and finally South: South-East and East part of the People's Republic of China.

Outbreaks with the largest number of dead animals were concentrated mainly in Bangladesh and China, where vaccinations have not been carried out in recent years.

The study of the correlation between the PPR incidence rate and the number of animals immunized in the most disadvantaged states found only modest reductions in incidence as a result of vaccination campaigns. Pearson's correlation coefficient was - 0.13 for Algeria, 0.19 for Bangladesh and -0.33 for Israel, which appears to be due to the small scale of vaccination campaigns. The obtained results of the epizootic analysis of PPR indicate insufficient coverage of animals susceptible to the causative agent of the disease by vaccination and the greater likelihood of new outbreaks of the disease not only in disadvantaged countries, but also the spread of infection in new territories.

\section{References}

1. F.I. Korennoj, Dudorova M.V., V.M. Gulenkin (Vladimir, FGU «VNIIZZH», 2010)

2. F.I. Korennoj (Diss. ... kand. geogr. nauk, Moskva, MGU, 2019)

3. L.P. Padilo, O.P. Biryukova, V.A. Agol'cov, Nauchnaya zhizn', 15, 7 (2020)

4. A.C. Banyard, S. Parida, C. Batten et al., J. Gen. Virol. (2010)

5. M. Baron, A. Diallo, R. Lancelot, Adv. Virus. Res. (2016)

6. O.A. Durojaiye, Trop. Anim. Hlth. Prod., 14 (1982)

7. EMPRES-i. Global Animal Disease Information System (2020)

8. C.W. Furley, Vet. Rec., 121 (1987)

9. S. Hodgson, et. al., Journal of Virology, 92 (2018)

10. Manual of Diagnostic Tests and Vaccines for Terrestrial Animals. Chapter 3.7.9. Peste des Petits Ruminants (Infection with Peste des Petits Ruminants Virus, 2019)

11. J.C. Mariner, Vaccine, 35 (2017)

12. A. Sen, P. Saravanan, V. Balamurugan, Expert Reviews Vaccines, 9, 7 (2010)

13. World Animal Health Information Database (WAHIS) Interface (2020) 